\title{
Analytical RF Pulse Heating Analysis for High Gradient Accelerating Structures
}

\author{
D. González-Iglesias, D. Esperante, B. Gimeno, Member, IEEE, M. Boronat, C. Blanch, N. Fuster-Martínez, \\ P. Martinez-Reviriego, P. Martín-Luna, J. Fuster
}

\begin{abstract}
The main aim of this work is to present a simple method, based on analytical expressions, for obtaining the temperature increase due to the Joule effect inside the metallic walls of an RF accelerating component. This technique relies on solving the 1D heat transfer equation for a thick wall, considering that the heat sources inside the wall are the ohmic losses produced by the RF electromagnetic fields penetrating into the metal with finite electrical conductivity. Furthermore, it is discussed how the theoretical expressions of this method can be applied to obtain an approximation to the temperature increase in realistic $3 \mathrm{D}$ RF accelerating structures, taking as an example the cavity of an RF electron photoinjector and a travelling wave linac cavity. These theoretical results have been benchmarked with numerical simulations carried out with a commercial Finite Element Method (FEM) software, finding good agreement among them. Besides, the advantage of the analytical method with respect to the numerical simulations is evidenced. In particular, the model could be very useful during the design and optimization phase of RF accelerating structures, where many different combinations of parameters must be analysed in order to obtain the proper working point of the device, allowing to save time and speed up the process. However, it must be mentioned that the method described in this manuscript is intended to provide a quick approximation to the temperature increase in the device, which of course is not as accurate as the proper $3 \mathrm{D}$ numerical simulations of the component.
\end{abstract}

Index Terms-RF pulse heating, thermal analysis, RF accelerating structures.

\section{INTRODUCTION}

RF pulse heating is a phenomenon by which metals are heated by the electric induced currents generated by a pulsed high power RF electromagnetic field. This heating has two different effects. The first one, which happens during the RF pulse, causes a superficial temperature increase within the RF skin depth range in a short time compared to the expansion mechanical time response of the material. Stress is induced on the metallic surface when the heating occurs faster than the expansion time of the material, which is governed by the speed of sound in it. Such material will suffer cyclic fatigue in which damage in the form of surface roughening and microcracks will occur over time if the induced stress is greater than the material elastic limit. According to recent studies of breakdown rates in high gradient linear accelerators, there is a direct correlation between these rates and RF pulse heating [1], [2]. Because of that, the reliable operating gradient could be potentially limited by the thermal stress suffered by these structures. The second effect occurs after the RF pulse

All the Authors are with the Instituto de Física Corpuscular (IFIC, UVCSIC), Valencia, Spain. ends and consists in the heat diffusion along the metallic slab and the temperature decrease in the surface until the next RF pulse. As a result, there is an overall temperature rise on the metallic walls of the cavity from one pulse until the arrival of the next. After many RF pulses the device temperature increases, the body expands and the resonant frequency shifts. This frequency shift will detune the cavities and might reflect the RF power.

This entails that the RF pulse heating effects must be taken into consideration when designing high gradient RF accelerating structures. There are several commercial codes (ANSYS [3], SIMULIA [4], MAFIA [5], etc.), based on Finite Differences in Time Domain (FDTD) or Finite Elements Method (FEM) [6], which allow a thermal analysis of the accelerating components. However, these software tools, which rely on numerical methods to solve the heat transfer equation to obtain the temperature distribution inside the component, usually have a considerable computational cost in terms of time and hardware in order to get accurate results. This fact can be even more problematic if a parametric study of different working points for the device is desired, in which many different simulations must be carried out.

To the best of the authors' knowledge, no analytical models can be found in the technical literature to estimate the average temperature increase in the metallic wall of an accelerator component after many RF pulses have been injected. With regard to the sharp temperature increase induced during the RF pulse, there is an approximate analytical formula that gives such magnitude at the metallic surface next to the RF fields [7]. However, this expression assumes an RF square pulse within the device, and is not valid for the Standing Wave (SW) structures where the square pulse is transformed into a transient one.

In this paper we describe a single procedure for analysing the RF pulse heating within a thick metallic wall exposed to an RF pulsed high power electromagnetic field is described. On the one hand, an exact analytical expression for obtaining the sharp temperature increase during the RF pulse is deduced. This is done for both a square RF pulse and a transient. On the other hand, an analytical formula for the average temperature increase in the wall after many pulses have been driven is provided. The procedure to reach such formulas is based on solving analytically the 1D heat transfer equation, which has the advantage of being much faster than any numerical method employed for solving such partial differential equation.

This paper is organized as follows. In Section II, the theoretical model employed for deriving analytical expressions for 
the thermal heating induced inside the component is discussed. In Subsection II-A analytical expressions for the temperature increase during the RF pulse are derived for the cases of a square RF pulse and for the SW structure in which the square pulse is transformed into a transient. In addition, numerical simulations with the commercial software Ansys Fluent [8] were also performed for benchmarking against the theoretical model. Next, in Subsection II-B, theoretical expressions are derived for the average temperature increase after many RF pulses. As in Subsection II-A, the model is verified by means of numerical simulations of the Ansys Thermal Transient (TTA) commercial software. Afterwards, in Sections III and IV the feasibility of using the 1D theoretical expressions for analysing the RF pulse heating phenomenon in $3 \mathrm{D}$ structures is explored by means of performing the thermal analysis of an RF photoinjector and a linac cavity. Finally, in Section V, the main conclusions of this study are outlined.

\section{THEORETICAL MODEL}

In this Section we present the theoretical background required to derive the analytical formulas that allow to describe the temperature increase inside the device wall as a function of time, caused by an electromagnetic RF pulsed signal. A typical RF pulsed signal consists in a gated harmonic signal. To analyse this phenomenon, the component wall is modelled as a metallic layer with thickness $L$ (see Fig. 1) that extends infinitely in the $y$ and $z$ dimmensions. The left side of the wall corresponds to the inner side of the device, i.e., where the RF electromagnetic fields are present. The right side of the wall is in contact with the cooling mechanism of the device, modelled as a convective heat exchange, which is characterized by its heat transfer coefficient $h$ and the fluid temperature $T_{\infty}$. Assuming the metal has a finite electrical conductivity $\sigma$, the RF electromagnetic fields inside the cavity (vacuum side in Fig. 1) penetrate into the conductor wall and are attenuated exponentially in a characteristic length $\delta$, known as the skin depth. Here, it is assumed that the RF electromagnetic field in the vacuum wall boundary has no dependece on the $y$ and $z$ coordinates. The time harmonic RF electromagnetic fields inside the metallic wall are given by [9]:

$$
\begin{gathered}
\overrightarrow{H_{\|}}(x, t)=H_{\|, 0} e^{-j \frac{x}{\delta}} e^{-\frac{x}{\delta}} e^{j \omega t} \hat{u}_{\|} \\
\vec{E}(x, t)=-\frac{1+j}{\delta \sigma}\left(\hat{x} \times \overrightarrow{H_{\|}}(x, t)\right) \\
\delta=\sqrt{\frac{2}{\sigma \mu_{0} \omega}}
\end{gathered}
$$

where $H_{\|, 0}$ is the amplitude of the parallel RF magnetic field at the interface, $x$ is the cartesian coordinate according to the reference scheme defined in Fig. $1, \hat{x}$ is the unitary vector along the $\mathrm{x}$ coordinate, $j=\sqrt{-1}$ is the imaginary unit, $\hat{u}_{\|}$is the unitary vector parallel to the surface, $\mu_{0}$ is the vacuum permeability, $\omega=2 \pi f$, and $f$ is the RF frequency. As it is well known, the RF electric field inside the metal will induce an electric current in the form $\vec{J}=\sigma \vec{E}$, which will dissipate an average power over an RF period $P_{d i s}$ due to the Joule effect [9]:

$$
P_{d i s}=\frac{1}{2} \operatorname{Re} \int_{V}\left(\vec{J} \cdot \overrightarrow{E^{*}}\right) d V
$$

where $\overrightarrow{E^{*}}$ represents the conjugate complex of the RF electric field phasor. Thus, the average power dissipated per unit area at a local position inside the wall and at a certain time during the RF pulse can be obtained as:

$$
P(x, t)=\frac{R_{s}}{\delta}\left|H_{\|, 0}(t)\right|^{2} e^{-\frac{2 x}{\delta}}
$$

where the surface resistance is $R_{s}=1 /(\delta \sigma)$, and it is assumed that the harmonic amplitude of the RF magnetic field varies with time $t$ as in fact occurs for an RF pulse, i.e., $H_{\|, 0}=$ $H_{\|, 0}(t)$. This dissipated power constitutes the heat source that increases the temperature of the component walls.

As a consequence of the symmetry of the slab in the $y-z$ plane, the RF electromagnetic fields only depend on the $x$ coordinate and the thermal problem is reduced to 1-D. Thus, the heat transfer equation that describes the thermal behaviour of the metallic layer for this 1D case can be written as follows [10]:

$$
\frac{\partial T}{\partial t}=\frac{\kappa}{\rho C_{e}} \frac{\partial^{2} T}{\partial x^{2}}+f(x, t)
$$

where $T$ is the temperature, $\kappa$ is the thermal conductivity, $\rho$ is the density, $C_{e}$ is the specific heat, and $f(x, t)$ represent the heat sources which, in our case, are related to the RF dissipated power in the form $f(x, t)=P(x, t) /\left(\rho C_{e}\right)$.

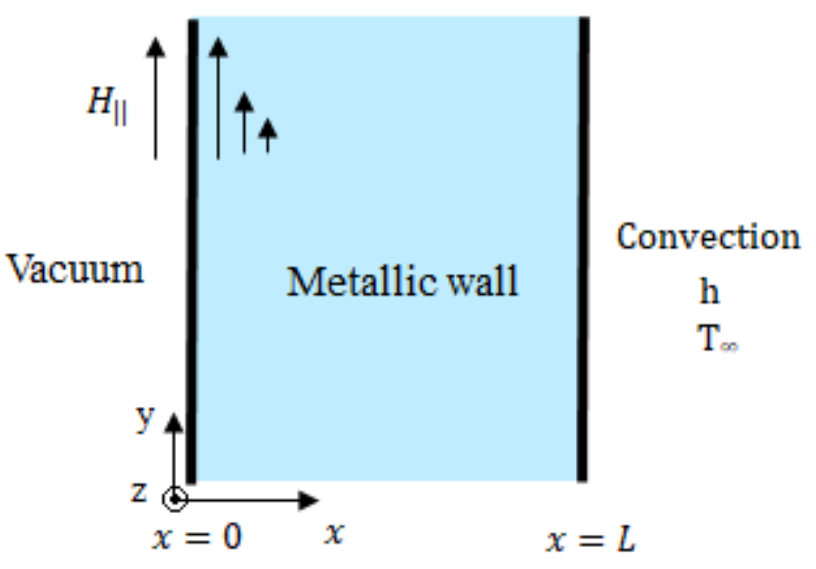

Fig. 1. Scheme of the device metallic wall. The inner surface $(x=0)$ corresponds to the vacuum side inside the component, the outer surface $(x=$ $L$ ) corresponds to the cooling side modelled as a convective heat exchange. The slab extends infinitely in the $y$ and $z$ directions.

The 1D heat transfer equation can be solved analytically as an infinite series in some cases. The particular form of the solution will vary depending on the initial conditions (spatial distribution of the temperature at the initial time) and the imposed boundary conditions. In the case we are 
interested in, the heat sources are time-dependent since the RF electromagnetic signal is composed by a succession of pulses with a repetition rate $f_{p}=1 / T_{p}$ ( $T_{p}$ being the pulse repetition period). During the pulse (characterized by $t_{o n}$ ), the electromagnetic fields oscillate harmonically in time, with an harmonic amplitude that also varies with time depending on the characteristic shape of the pulse. When the pulse ends there is a time lapse in which there are no heat sources in the wall until the start of the next one. In this work, two pulse shapes will be taken into consideration, namely, the square pulse (see Fig. 2), and the pulse with transient (see Fig. 3), which is the transformation of the square pulse in a SW structure.

\section{A. Temperature increase during the pulse}

First, we will focus on obtaining the temperature increase during the RF pulse. The typical values of $t_{\text {on }}$ in pulsed RF accelerators is usually within the range from several hundred nanoseconds to a few microseconds. These time lapses are shorter than the typical times that takes the diffusion mechanism to transfer the heat across the wall from the vacuum side to the cooling boundary. Afterwards, this point will be demonstrated (see Example II) by means of solving numerically the heat transfer equation, using a set of parameters within the typical range for RF accelerating devices. Since a negligible amount of heat is transferred across the wall during the RF pulse, the heat exchange between the convection medium and the right wall boundary will affect the temperature only in the neighbourhood of such boundary. If we are interested in obtaining the temperature increase during the pulse, which only is noticeable in a length of a few skin depths from the vacuum wall, the convection mechanism of the right wall can be dropped from the calculations by assuming a thermally isolated wall, i.e., $h=0 \mathrm{Wm}^{-2} \mathrm{~K}^{-1}$. This assumption simplifies the mathematical problem of solving the heat transfer equation, which allows to obtain a simple analytical expression for the temperature increase during the pulse. Next, the procedure to solve the $1 \mathrm{D}$ heat transfer equation, assuming that the right boundary is thermally isolated, is detailed.

Hence, in this case both sides of the metallic wall are assumed to be thermally isolated, i.e., no heat exchange occurs between the body under study and the environment. This assumption implies that the radiative heat exchange mechanism is neglected in this study. This approximation can be done since the heat source term due to the RF losses is much larger than the power radiated, for the typical temperature values of the wall cavities that are of interest in the area of RF accelerators. Mathematically, this imposes the following boundary conditions to the eq. (3):

$$
\left.\frac{\partial T}{\partial x}\right|_{x=0}=0,\left.\quad \frac{\partial T}{\partial x}\right|_{x=L}=0
$$

With regard to the initial conditions, an uniform temperature $T_{0}$ along the wall is assumed. The last information required to solve the differential equation is to specify the temporal dependence of the RF pulse, i.e., to define the time variation of the harmonic envelope of the RF magnetic field at the vacuum wall: $H_{\|, 0}(t)$. Next, the heat transfer equation will be solved assuming two typical different RF pulse profiles. The first case is a flat square pulse with length $t_{o n}$. This case matches well with the operation in a Travelling Wave (TW) structure. The second case will be a flat square pulse of duration $t_{\text {on }}$ feeding a Standing Wave (SW) structure. Here, there is a rising field in the cavity, that depends on the cavity filling time, until the $\mathrm{RF}$ electromagnetic field amplitude reaches its maximum value [11].

Hence, the following differential equation with the previous boundary conditions needs to be solved:

$$
\frac{\partial T}{\partial t}=D^{2} \frac{\partial^{2} T}{\partial x^{2}}+f(x, t)
$$

where $D^{2}=\frac{\kappa}{\rho C_{e}}$. Let us assume that the temperature can be described as the sum of two auxiliary functions, $v(x, t)$ and $w(x, t)$ :

$$
\begin{gathered}
T(x, t)=v(x, t)+w(x, t) \\
\left.\frac{\partial v}{\partial x}\right|_{x=0}=0,\left.\quad \frac{\partial v}{\partial x}\right|_{x=L}=0, \quad v(x, 0)=0 \\
\frac{\partial w}{\partial t}=D^{2} \frac{\partial^{2} w}{\partial x^{2}} \\
\left.\frac{\partial w}{\partial x}\right|_{x=0}=0,\left.\quad \frac{\partial w}{\partial x}\right|_{x=L}=0, \quad w(x, 0)=T(x, 0)
\end{gathered}
$$

First, we solve the partial differential equation for $w(x, t)$. If we try the following variable separation: $w(x, t)=U(t) X(x)$, the partial differential equation becomes uncoupled and a set of two differential equations is obtained:

$$
\begin{array}{r}
\frac{d^{2} X}{d x^{2}}+\lambda X=0 \\
\frac{d U}{d t}+D^{2} \lambda U=0
\end{array}
$$

where $\lambda$ is a constant. The solution of the differential equation given by eq. (6) is well known [12] and, after imposing the boundary conditions to the derivatives, the following possible values for $\lambda$ are found: $\lambda_{k}=\left(\frac{\pi k}{L}\right)^{2}$, being $k=0,1,2, .$. a positive integer number. Thus, it is reached $X_{k}(x)=s_{k} \cos \left(\frac{\pi k}{L} x\right)$, where $s_{k}$ is a coefficient. Similarly, the solution for eq. (7) is straightforward and arises $U_{k}(t)=d_{k} e^{-\left(\frac{\pi D k}{L}\right)^{2} t}$, where $d_{k}$ is a coefficient. Combining both solutions, the function $w(x, t)$ can be written as an infinite linear combination of the possible values of $\lambda$ :

$$
w(x, t)=\sum_{n=0}^{+\infty} a_{n} \cos \left(\frac{\pi n}{L} x\right) e^{-\left(\frac{\pi D n}{L}\right)^{2} t}
$$

where $a_{n}=s_{n} d_{n}$.

Finally, the initial conditions for $w(x, t)$ at $t=0$ are imposed, which correspond to the initial temperature distribution, i.e., $w(x, 0)=T(x, 0)$. Now, it remains to solve the differential equation for $v(x, t)$. Taking a look at the solution for $w(x, t)$, we will try a solution of this kind: 


$$
v(x, t)=\sum_{n=0}^{+\infty} u_{n}(t) \cos \left(\frac{\pi n}{L} x\right)
$$

Substituting this expression in the differential equation, it leads:

$$
\sum_{n=0}^{+\infty}\left[\frac{d u_{n}}{d t}+\left(\frac{\pi D n}{L}\right)^{2} u_{n}\right] \cos \left(\frac{\pi n}{L} x\right)=f(x, t)
$$

We can make the ansatz that the heat sources can be split in the following form: $f(x, t)=g(x) h(t)$. For the physical problem under consideration, the heat source term is obtained from the RF power losses given by eq. (2), which is in fact compatible with the above assumption. The $g(x)$ can be developed as a Fourier series:

$$
g(x)=\frac{g_{0}}{2}+\sum_{n=1}^{+\infty} g_{n} \cos \left(\frac{\pi n}{L} x\right)
$$

where the coefficients $g_{n}$ are given by

$$
g_{n}=\frac{2}{L} \int_{0}^{L} g(x) \cos \left(\frac{\pi n}{L} x\right) d x, \quad g_{0}=\frac{2}{L} \int_{0}^{L} g(x) d x
$$

Substituting this development of the heat transfer function into eq. (8), we reach:

$$
\begin{aligned}
& \frac{d u_{0}}{d t}=\frac{g_{0}}{2} h(t) \\
& \frac{d u_{n}}{d t}+\left(\frac{\pi D n}{L}\right)^{2} u_{n}=g_{n} h(t), \quad n \geqslant 1
\end{aligned}
$$

The solution for both eqs. (9) and (10) results in an homogeneous solution and a particular solution. Homogeneous solutions are straightforward in both cases, whilst the particular solutions depend on the specific form that $h(t)$ takes. When the functions $u_{n}(t)$ are known, the integration constant is determined by imposing the initial condition $u_{n}(0)=0$, which is derived from $v(x, 0)=0$.

Finally, according to eq. (5), the temperature within the wall is obtained by combining the solutions of $v(x, t)$ and $w(x, t)$.

Next, the solution of the heat transfer differential equation is provided for the square pulse and the pulse with transient cases.

1) Square pulse: The harmonic amplitude of a square pulse of duration $t_{o n}$ is described as:

$$
H_{\|, 0}(t)= \begin{cases}H_{\|, 0}, & 0 \leqslant t \leqslant t_{\text {on }} \\ 0, & t>t_{\text {on }}\end{cases}
$$

In Fig. 2 the scheme of a sequence of square pulses is shown. By solving the eq. (4) for a single square RF pulse, the induced temperature increase is given by:

$$
\begin{array}{r}
\Delta T(x, t)=g_{0} t+\sum_{n=1}^{\infty} \frac{g_{n}}{\left(\frac{\pi D n}{L}\right)^{2}}\left(1-e^{-\left(\frac{\pi D n}{L}\right)^{2} t}\right) \cos \left(\frac{\pi n x}{L}\right), \\
0 \leqslant t \leqslant t_{\text {on }}
\end{array}
$$

$$
\begin{aligned}
& \Delta T(x, t)=g_{0} t_{\text {on }}+ \\
& \sum_{n=1}^{\infty} \frac{g_{n}}{\left(\frac{\pi D n}{L}\right)^{2}}\left(1-e^{-\left(\frac{\pi D n}{L}\right)^{2} t_{o n}}\right) e^{-\left(\frac{\pi D n}{L}\right)^{2}\left(t-t_{o n}\right)} \cos \left(\frac{\pi n x}{L}\right) \\
& , t>t_{\text {on }} \\
& \quad \text { with }
\end{aligned}
$$

$$
\begin{gathered}
g_{0}=\frac{2 \alpha}{L}\left(1-e^{-\frac{2 L}{\delta}}\right) \\
g_{n}=\frac{8 \alpha L}{4 L^{2}+\pi^{2} \delta^{2} n^{2}}\left(1-e^{-\frac{2 L}{\delta}}(-1)^{n}\right) \\
\alpha=\frac{R_{s}\left|H_{||, 0}\right|^{2}}{2 \rho C_{e}} ; \quad D=\sqrt{\frac{\kappa}{\rho C_{e}}}
\end{gathered}
$$

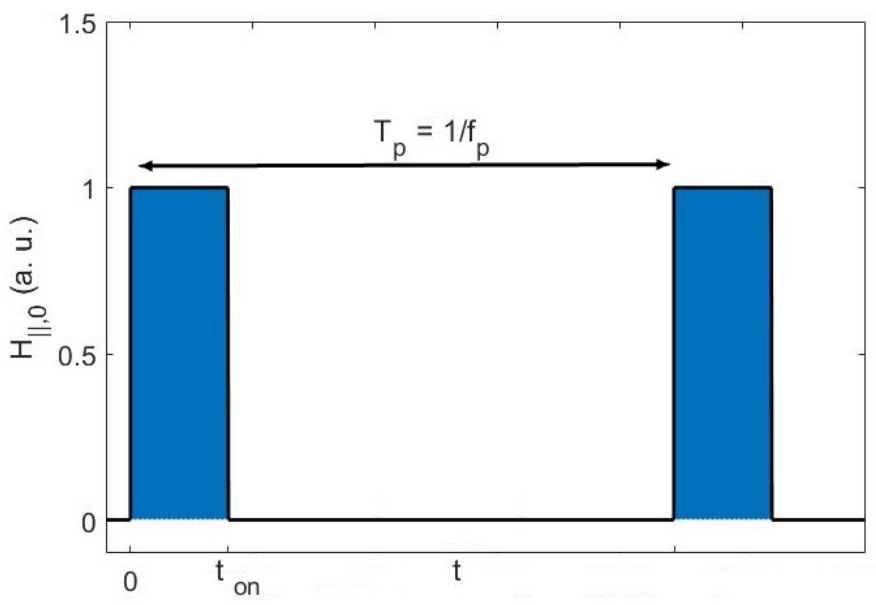

Fig. 2. Scheme of pulsed signal consisting of a train of square pulses. The pulse has a length of $t_{o n}$ and the pulse repetition period is $T_{p}$ (with a repetition frequency of $f_{p}$ ).

2) Pulse with transient: Let us assume that the RF generator provides a square pulse (similar to eq. (11)) to the RF circuit. For a SW cavity, such square pulse will be transformed into a transient given by the following expressions [11]:

$$
H_{\|, 0}(t)= \begin{cases}H_{\|, 0}\left(1-e^{-\frac{t}{\tau}}\right), & 0 \leqslant t \leqslant t_{\text {on }} \\ H_{\|, 0}\left(1-e^{-\frac{t_{\text {on }}}{\tau}}\right) e^{-\frac{t-t_{\text {on }}}{\tau}}, & t>t_{\text {on }}\end{cases}
$$

where $\tau$ is the filling time of the cavity. In Fig. 3 it is shown the scheme of a sequence of pulses with transient.

After solving the eq. (4) for one single transient pulse, the following expressions for the temperature increase arise:

$$
\Delta T(x, t)=u_{0}(t)+\sum_{n=1}^{\infty} u_{n}(t) \cos \left(\frac{\pi n x}{L}\right), \quad 0 \leqslant t \leqslant t_{\text {on }}
$$

$$
\begin{aligned}
& \Delta T(x, t)=u_{0}\left(t_{\text {on }}\right)+\frac{1}{2} v_{0}\left(t-t_{\text {on }}\right)+ \\
& \sum_{n=1}^{\infty}\left(v_{n}\left(t-t_{\text {on }}\right)+u_{n}\left(t_{\text {on }}\right) e^{-\left(\frac{\pi D n}{L}\right)^{2}\left(t-t_{\text {on }}\right)}\right) \cos \left(\frac{\pi n x}{L}\right) \\
& , \quad t>t_{\text {on }}
\end{aligned}
$$


with

$$
\begin{gathered}
u_{0}(t)=\frac{g_{0}}{2}\left[t+\tau\left(2 e^{-\frac{t}{\tau}}-\frac{1}{2} e^{-\frac{2 t}{\tau}}-\frac{3}{2}\right)\right] \\
u_{n}(t)=\frac{g_{n}}{\left(\frac{\pi D n}{L}\right)^{2}}\left(1-e^{-\left(\frac{\pi D n}{L}\right)^{2} t}\right)+ \\
\frac{g_{n}}{\left(\frac{\pi D n}{L}\right)^{2}-\frac{2}{\tau}}\left(e^{-\frac{2 t}{\tau}}-e^{-\left(\frac{\pi D n}{L}\right)^{2} t}\right) \\
+\frac{2 g_{n}}{\left(\frac{\pi D n}{L}\right)^{2}-\frac{1}{\tau}}\left(e^{-\left(\frac{\pi D n}{L}\right)^{2} t}-e^{-\frac{t}{\tau}}\right) \\
v_{n}(t)=\frac{g_{n}\left(1-e^{-\frac{t_{o n}}{\tau}}\right)^{2}}{\left(\frac{\pi D n}{L}\right)^{2}-\frac{2}{\tau}}\left[e^{-\frac{2 t}{\tau}}-e^{-\left(\frac{\pi D n}{L}\right)^{2} t}\right]
\end{gathered}
$$

where $g_{0}$ and $g_{n}$ are the same as in eqs. (13a)-(13b).

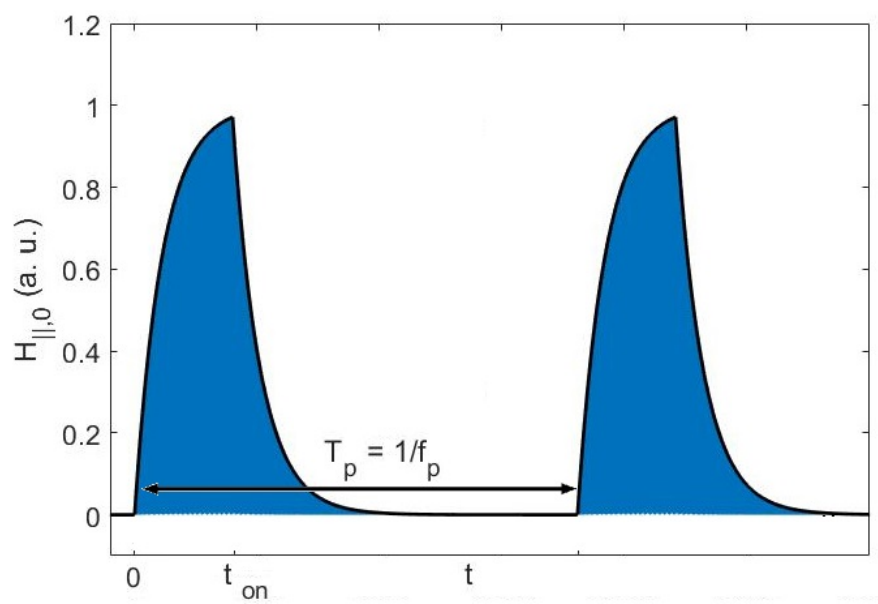

Fig. 3. Scheme of pulsed signal consisting of a train of pulses with transient The pulse has a length of $t_{\text {on }}$ (as it is seen by the RF power source) and the pulse repetition period is $T_{p}$ (with a repetition frequency of $f_{p}$ ).

Now, these deduced expressions can be applied to study the temperature increase during the RF pulse. First, the results provided by the theoretical formulas are shown for a particular case (Example I), and the theory is benchmarked with the Ansys Fluent numerical simulations. In the Example II it is demonstrated that, for the typical range of parameters of the $\mathrm{RF}$ accelerators, the temperature increase near the vacuum wall during the RF pulse is not affected by the presence of a cooling mechanism in the opposite wall of the device. Finally, in Example III, the maximum temperature increase in the wall is obtained as a function of the RF pulse length in the case with transient. Furthermore, a comparison between the results provided by the analytic theory and the approximate expression given in [7] (see eq. (3.38), in pag. 99) for estimating the RF pulse heating is presented.

Example I: The heating of the wall will be examined for an RF pulse with transient. It is assumed that the right side is thermally isolated ( $\left.h=0 W^{-2} K^{-1}\right)$, and the initial temperature distribution is uniform. The wall material is copper. The simulation parameters are: $t_{\text {on }}=400 \mathrm{~ns}, \tau=$
$112.5 \mathrm{~ns} \mathrm{f}_{\mathrm{p}}=400 \mathrm{~Hz}, \mathrm{H}_{\|}=405200 \mathrm{~A} / \mathrm{m}, \delta=0.595 \mu \mathrm{m}$, $L=1 \mathrm{~mm}, \kappa=401 \mathrm{Wm}^{-1} \mathrm{~K}^{-1}, \rho=8940 \mathrm{~kg} / \mathrm{m}^{3}$, $C_{e}=376.818 \mathrm{~J} \mathrm{~kg}^{-1} \mathrm{~K}^{-1}$. All the previous parameters (with the exception of $L$ ) correspond to a preliminary design of an X-band photoinjector cavity operating with $200 \mathrm{MV} / \mathrm{m}$ cathode field at $f=11.994 \mathrm{GHz}$ [13].

Since the copper skin depth value is much smaller than the slab length, a multiple mesh with two different sizes has been employed for the Ansys Fluent simulations. A fine mesh is used in the region where the heat sources are present, i.e., from the left boundary up to $12 \mu m(\approx 20 \delta)$ and a coarser one in the rest of the slab. With regard to the step size, a fixed value of $10 \mu \mathrm{m}$ was chosen for the coarse mesh region and three different values were tested for the fine mesh: $0.06 \mu \mathrm{m}, 0.1 \mu \mathrm{m}$, and $0.5 \mu \mathrm{m}$. The choice of different step sizes was aimed at testing the convergence of the Ansys Fluent simulations. The final time of the thermal simulations is $t_{\text {end }}=2000 \mathrm{~ns}$. The time of computation required to run each of the above Ansys Fluent simulations ranged from 10 to 12 minutes, depending on the step size, employing a standard PC (Intel Core i5, $3.40 \mathrm{GHz}, 8 \mathrm{~GB}$ RAM). Hence, the total computational time required to analyse the temperature increase within the slab is approximately 32 minutes.

It is interesting to compare the Ansys Fluent simulations computational times with the time required to calculate the analytical solution, which is provided by the eqs. (15a) and (15b). For the analytical solution, the convergence of the sum can be checked by trying different number of terms in the sum. The results of this test are presented in Fig. 4, where the absolute temperature difference between the cases with $N$ terms considered in the sum and the case with $10^{5}$ terms is shown. It can be noticed that with the lower number of sum terms considered, namely $N=10000$, the difference with respect to $N=100000$ is the range of $10^{-19}{ }^{\circ} C$, which proves the convergence of the analytical sum even with the lower number of terms considered. Regarding the computational times of the analytical solution, these are summarized in the Table I.

TABLE I

ANALYTICAL SOLUTION COMPUTATIONAL TIMES

\begin{tabular}{|c|c|}
\hline $\mathrm{N}$ & Computational time (s) \\
\hline 10000 & 0.4 \\
\hline 20000 & 0.8 \\
\hline 50000 & 2.1 \\
\hline 100000 & 4.1 \\
\hline
\end{tabular}

The computational time required for the thermal analysis employing the analytical solution is around seven seconds, whilst the time needed for the Ansys Fluent computation was a bit more than half an hour. This means that the analytical solution is more than 200 times faster than the Ansys Fluent analysis. Hence, it becomes evident the huge improvement in terms of computational time that is achieved by using the analytical formula compared to the numerical solutions provided by Ansys Fluent.

Now, we can compare the temperature increase in the slab obtained with the analytical formula and with Ansys Fluent, 


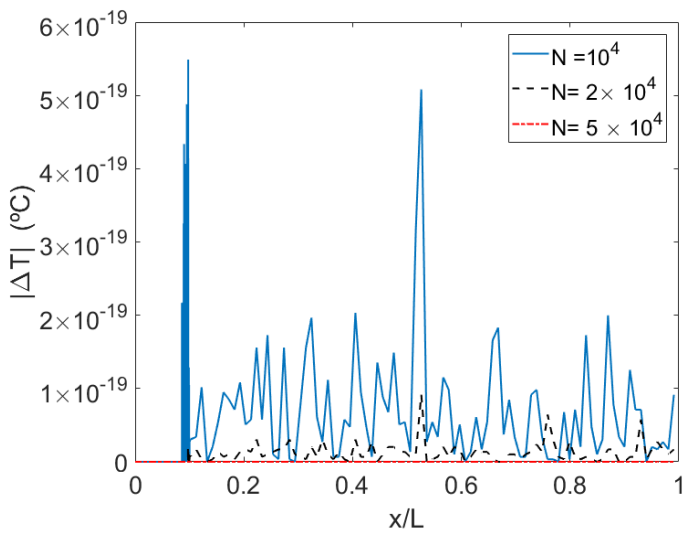

Fig. 4. Absolute temperature difference between the analytical solution with different number of terms in the sum $(N)$ and the analytical solution with $N=10^{5}$. These results belong to the slab temperature along the wall depth, for a time of $t=2000 \mathrm{~ns}$.

which are summarized in Fig. 5. In Fig. 5(a) it is depicted the temperature variation with time at the vacuum wall end $(x=0)$, whilst in Fig. 5(c) it is shown the spatial variation of the wall temperature at the end of the simulation time $\left(t_{\text {end }}=\right.$ 2000 ns). In Figs. 5(b) and 5(d) show the absolute temperature difference between the Ansys Fluent and the analytical results of Figs. 5(a) and 5(c), respectively, for a better comparison. In addition, the chi-squared statistical test is performed to give a quantitative view of the concordance or disagreement between the analytical and the numerical results [14]. For the data of Fig. 5(a), it is found a reduced chi-squared of $\tilde{\chi}_{0}^{2}=0.0012$, the probability of a $\tilde{\chi}^{2}$ equal or greater than $\tilde{\chi}_{0}^{2}$, is $P\left(\tilde{\chi}^{2} \geqslant\right.$ $\left.\tilde{\chi}_{0}^{2}\right) \approx 100 \%$. Similarly, for the data of Fig. 5(c), we found that $\tilde{\chi}_{0}^{2}=0.014$ and $P\left(\tilde{\chi}^{2} \geqslant \tilde{\chi}_{0}^{2}\right) \approx 100 \%$. According to [14], values of $P\left(\tilde{\chi}^{2} \geqslant \tilde{\chi}_{0}^{2}\right)<5 \%$ indicate significant disagreement between the data. Hence, values of $P\left(\tilde{\chi}^{2} \geqslant \tilde{\chi}_{0}^{2}\right) \geqslant 5 \%$ will indicate acceptable or good match. For a qualitative description of the agreement based on the chi-squared statistics, we define the following criterion: when $5 \% \leqslant P\left(\tilde{\chi}^{2} \geqslant \tilde{\chi}_{0}^{2}\right)<60 \%$ we will consider acceptable match or concordance, whilst if $P\left(\tilde{\chi}^{2} \geqslant \tilde{\chi}_{0}^{2}\right) \geqslant 60 \%$ we will talk about good concordance. According to this, in the plots of Fig. 5 a good concordance is found among the analytical results (eqs. 15a and 15b) and the Ansys Fluent calculations.

Example II: In this case, the heating of the wall will be examined for an RF pulse with transient under two different scenarios: with and without the cooling system. The cooling system is assumed to be in contact with the right wall surface and it is modelled as a convection heat exchange mechanism described by the heat transfer coefficient of the fluid, $h$, and its temperature, $T_{\infty}$. For this example we take $T_{\infty}=0{ }^{\circ} \mathrm{C}$ and $h=1.2 \times 10^{4} \mathrm{Wm}^{-2} \mathrm{~K}^{-1}$, where the value of $h$ corresponds to a typical value for a water cooling channel in $\mathrm{RF}$ accelerators [7]. The remaining parameters are the same as in Example I.

In Fig. 6, the temperature increase along the metallic wall is depicted at a certain time after the end of the pulse $(t=2000 \mathrm{~ns})$. No significant differences are found between the numerical simulations with Ansys Fluent for the case with
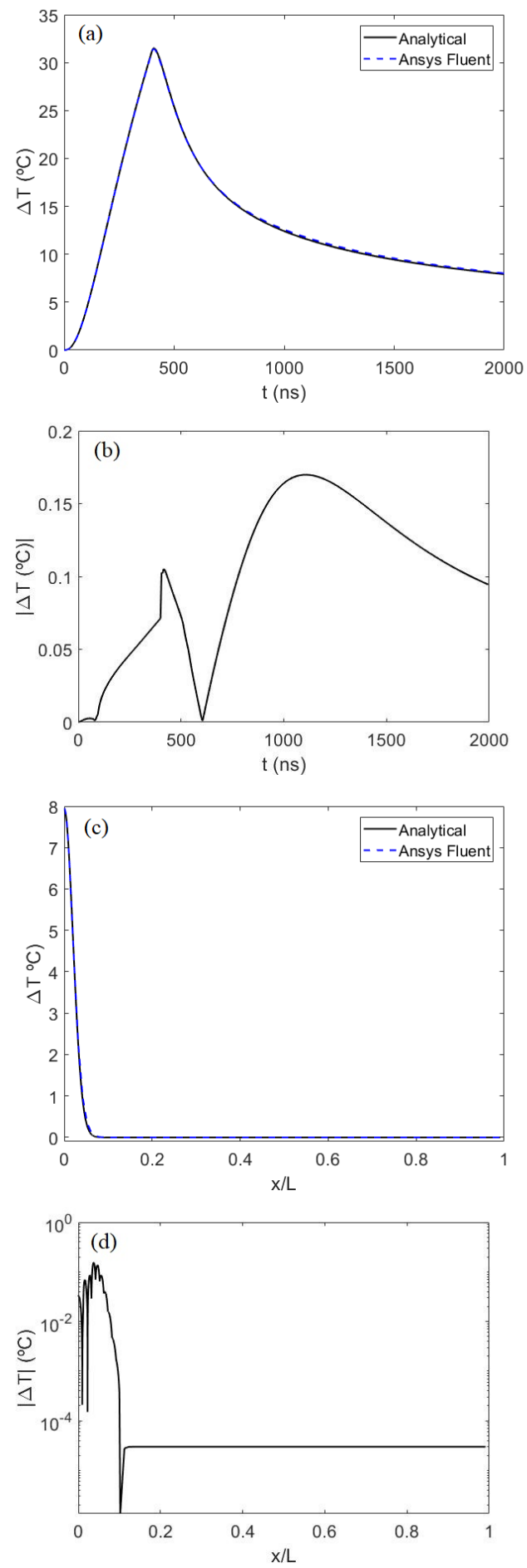

Fig. 5. (a) Temperature increase at the left wall surface $(x=0)$ as a function of time. (b) Absolute temperature difference between the analytical model and the ANSYS Fluent results shown in a). (c) Temperature increase as a function of the depth in the wall, for $t=2000 \mathrm{~ns}$.(d) Absolute temperature difference between the analytical model and the ANSYS Fluent results shown in (c).

cooling and the analytical expression that assumes perfect heat isolation. In fact, the chi-squared test analysis gives $\tilde{\chi}_{0}^{2}=0.014$ and $P\left(\tilde{\chi}^{2} \geqslant \tilde{\chi}_{0}^{2}\right) \approx 100 \%$. Therefore, a good agreement between both cases is found. Very similar results are obtained when comparing the temperature increase at the 
left wall surface $(x=0)$ as a function of time. This plot, that is analogous to Fig. 5(a), has been omitted for the sake of brevity.

At the view of the results, it has been demonstrated that, for a time lapse up to several times the RF pulse length, there is no significant difference in the temperature increase between the case in which the right wall side is cooled and the case with the right boundary thermally isolated. As argued before, the pulse durations in RF accelerator systems are too short to allow effective heat diffusion across the wall and the heat remains focused on the neighbourhood of the heat sources region, i.e. the vacuum wall. Moreover, for this example the wall depth is $L=1 \mathrm{~mm}$, which is smaller than the typical wall depths employed in realistic devices that can span up to several centimetres. Hence, if the previous statement is true for $L=1 \mathrm{~mm}$ it will also be valid for higher values of $L$. As it has been evidenced, the theoretical model presented in Subsection II-A is able to provide an accurate estimation of the temperature increase in the wall, even when there is a cooling system.
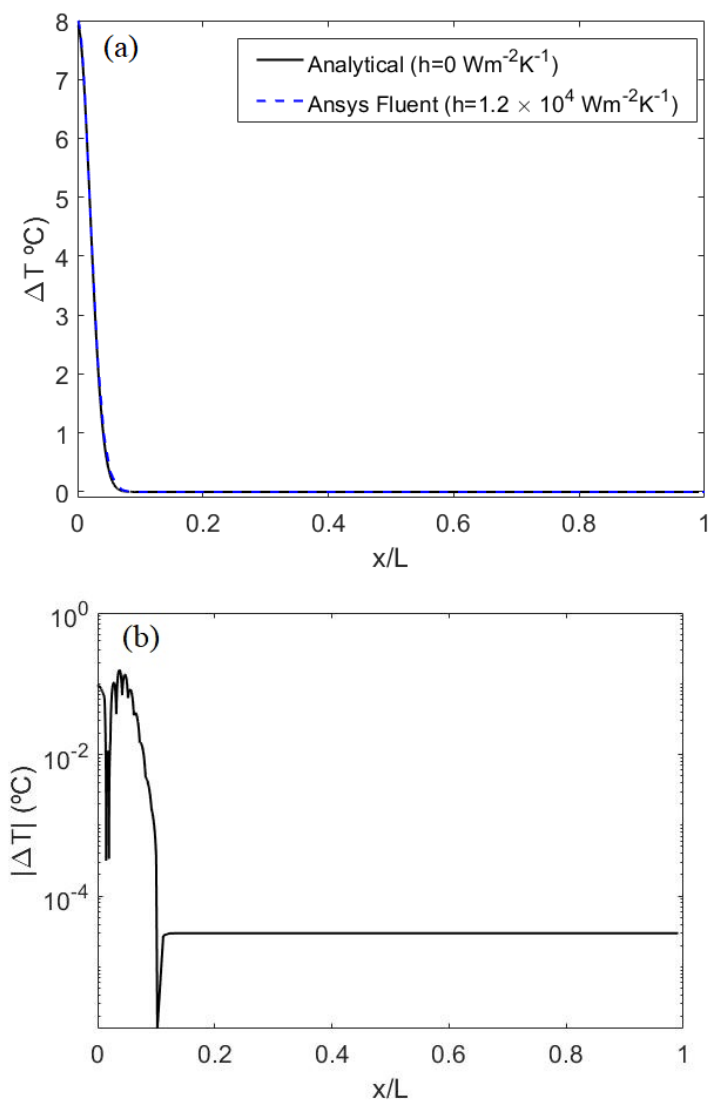

Fig. 6. (a) Temperature increase as a function of the wall depth for $t=$ 2000 ns under two scenarios: with and without water cooling system. (b) Absolute temperature difference between the analytical and ANSYS Fluent cases depicted in (a).

Example III: Here, the maximum temperature increase in the wall during the RF pulse for the case with transient is analysed using the analytical formulas presented in Subsection II-A. The same parameters as in Example I are chosen, with the exception of $L=20 \mathrm{~mm}$. As previously argued, the vacuum wall boundary is the region where the highest temperature rise is expected. In Fig. 7 the temperature increase at the vacuum wall side as a function of time is depicted for a pulse length of $t_{o n}=400 \mathrm{~ns}$. As expected, the maximum temperature increase takes place when the RF power level is maximum, corresponding with the time at which the RF source is switched off $\left(t=t_{\text {on }}\right)$.

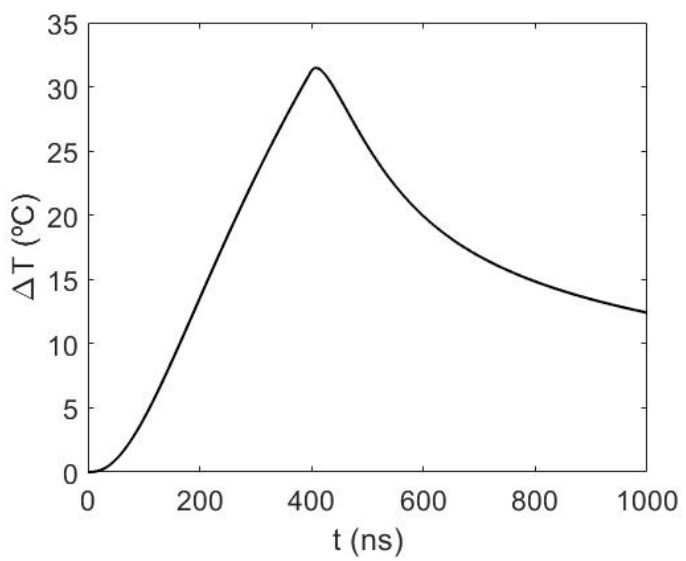

Fig. 7. Temperature increase at $x=0$ as a function of time, for $t_{o n}=$ 400 ns.

Next, the maximum temperature increase for different values of the RF pulse duration is investigated. This is done in Fig. 8, where such maximum temperature increase is depicted using the analytical formulas for the cases with and without transient. Besides, the results provided by the approximate expression for the pulse heating using square RF pulses in Ref. [7] are also included in the plot. In this figure, a meaningful difference in the temperature increase between the case with and without transient is evidenced. It is also shown that the formula in Ref. [7] gives good predictions for the case without transient, since the exact analytical formula for that case is similar to the results obtained with the formula in Ref. [7]. However, using the formula in Ref. [7] for a case with transient will not provide an accurate solution, since this formula was not intended for such case (of course, it is possible to use the theory in Ref. [7] to obtain the proper temperature increase by evaluating numerically the Green's functions). At the view of the results, it has been shown the utility of using the accurate analytical expressions presented in this article (eq. (15a) and (15b)) for estimating the RF pulse heating during the pulse when there is a transient.

\section{B. Average temperature increase after many $R F$ pulses}

Once the temperature increase during the RF pulse is known, the next step is to obtain the average temperature increase in the wall after many RF pulses have been driven through the device. In order to do this, the heat transfer equation is analytically solved under two assumptions that simplify the mathematical procedure, allowing to obtain simple formulas that can be easily applied. First, the heat sources in the wall given by eq. (2) are replaced by an equivalent superficial heat flux entering through the vacuum wall. This 

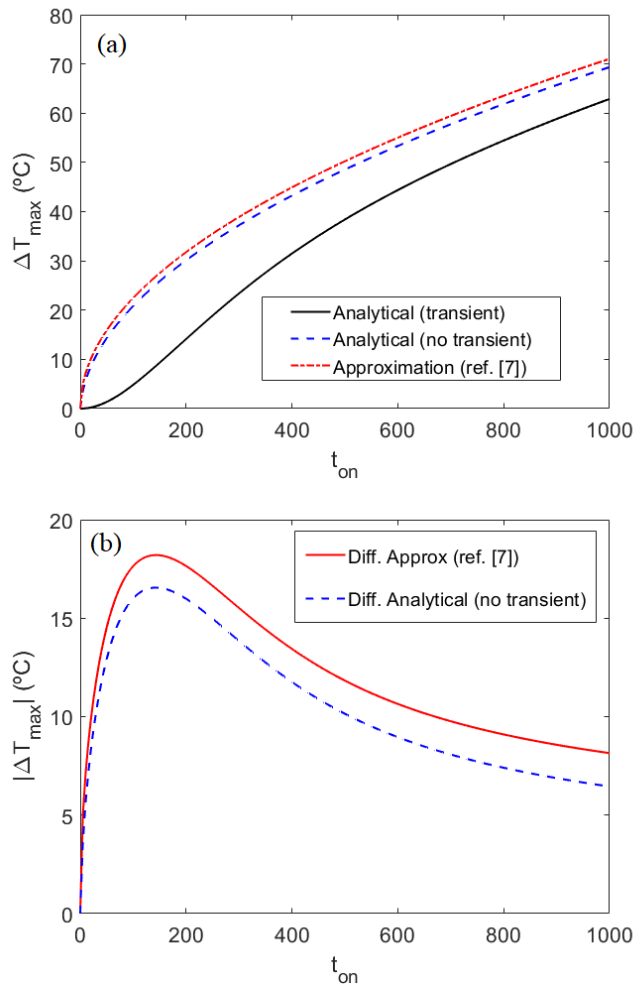

Fig. 8. (a) Maximum temperature increase at the wall as a function of the RF pulse length. (b) Absolute temperature difference between the analytical with transient and the analytical without transient/approximation in Ref. [7] cases depicted in (a).

equivalent superficial heat flux is obtained by integrating the total contribution of eq. (2) along the wall depth. This assumption gives good results since the skin depth values in $\mathrm{RF}$ accelerators is much shorter than the wall depth and hence the volume distribution of the heat sources is focused on the neighbourhood of the vacuum wall. The second assumption considers that the temporal variation of the heat source can be replaced by a time-constant heat source with the time-average value of the heat generated along the RF pulse sequence. For this case, the presence of the cooling system cannot be neglected in the calculations and it is taken into account. The differential heat transfer equation and its corresponding boundary conditions take the form:

$$
\begin{gathered}
\frac{\partial T}{\partial t}=D^{2} \frac{\partial^{2} T}{\partial x^{2}} \\
\left.\frac{\partial T}{\partial x}\right|_{x=0}=-\frac{q_{s, 0}}{\kappa},\left.\quad \frac{\partial T}{\partial x}\right|_{x=L}=-\frac{h}{\kappa}\left[T(L, t)-T_{\infty}\right]
\end{gathered}
$$

with

$$
q_{s, 0}=\frac{1}{t_{\text {end }}} \int_{0}^{t_{\text {end }}} d t \int_{0}^{L} P(x, t) d x
$$

where $t_{\text {end }}$ is the time that spans the RF pulsed sequence. The procedure to solve analytically such differential equation is very similar to the described previously in Subsection II-A and it will be omitted here for the sake of brevity. Thus, the average temperature along the wall as a time function is given by the following expressions, assuming as initial conditions a uniform temperature along the wall $T_{0}$ :

$$
\begin{gathered}
T(x, t)=T_{\infty}+\frac{q_{s, 0}}{\kappa}\left(L+\frac{\kappa}{h}\right)-\frac{q_{s, 0}}{\kappa} x \\
+\sum_{n=1}^{\infty} C_{n} \cos \left(\lambda_{n} x\right) e^{-\left(D \lambda_{n}\right)^{2} t} \\
C_{n}=\frac{\frac{1}{\lambda_{n}} \sin \left(\lambda_{n} L\right)\left[T_{0}-T_{\infty}^{\prime}\right]-\frac{q_{s, 0}}{\lambda_{n}^{2} \kappa}\left[1-\left(1+\frac{h L}{\kappa}\right) \cos \left(\lambda_{n} L\right)\right]}{\frac{L}{2}+\frac{\sin 2 \lambda_{n} L}{4 \lambda_{n}}}
\end{gathered}
$$

where

$$
\begin{gathered}
T_{\infty}^{\prime}=T_{\infty}+\frac{q_{s, 0}}{\kappa}\left(L+\frac{\kappa}{h}\right) \\
\lambda_{n} \tan \left(\lambda_{n} L\right)=\frac{h}{\kappa}
\end{gathered}
$$

Eq. (17) has to be solved numerically to obtain the eigenvalues $\lambda_{n}$ that allow us to get the coefficients $C_{n}$. As it can be noticed in eq. (16), the function dependence on time is of the form $e^{-\left(D \lambda_{n}\right)^{2} t}$. Thus, for a sufficiently long time the temperature in the wall will reach a steady state distribution given by

$$
T_{\text {steady }}(x)=T_{\infty}+\frac{q_{s, 0}}{\kappa}\left(L+\frac{\kappa}{h}\right)-\frac{q_{s, 0}}{\kappa} x
$$

Next, in Example IV the theoretical results provided by eq. (16) for the average temperature in the wall, and the results provided by the Thermal Transient Ansys (TTA) numerical simulations [3], are presented for benchmarking between them.

Example IV: For this case, the same parameters as in Example III are taken, with the exception of $L=15 \mathrm{~mm}$. The average temperature increase of the wall will be examined after many RF pulses have been driven throw the device and the steady state has been achieved. In Fig. 9(a) the average temperature increase at the vacuum side of the wall (i.e., $x=0$ ) is depicted as a function of time for the analytical formula (eq. (16)) and the TTA simulations. In Fig. 9(b) the absolute temperature difference between the analytical and the TTA results is shown. The results for the chi-squared test are $\tilde{\chi}_{0}^{2}=0.707$ and $P\left(\tilde{\chi}^{2} \geqslant \tilde{\chi}_{0}^{2}\right)=98.41 \%$. Thus, there is a good agreement between both data series.

Similarly, the average temperature increase along the wall at the steady state is depicted in Fig. 10(a). For comparison, in Fig. 10(b) the absolute temperature difference between the analytical and the TTA data is shown. In this case, the chi-squared test also outcomes a good match with values of $\tilde{\chi}_{0}^{2}=6.56 \times 10^{-6}$ and $P\left(\tilde{\chi}^{2} \geqslant \tilde{\chi}_{0}^{2}\right) \approx 100 \%$. It is worth mentioning that the linear variation of the temperature along the wall predicted at the steady state by eq. (18) is indeed observed in the plot.

Regarding the computational time, 7 minutes were required to get the results from the TTA simulations in an Intel Core i5, $3.40 \mathrm{GHz}$, with $8 \mathrm{~GB}$ RAM. For the analytical solution, firstly the eigenvalues given by eq. (17) must be obtained. For this purpose, we employed the Matlab routine $f$ zero that solves the roots of nonlinear functions. This method was able 

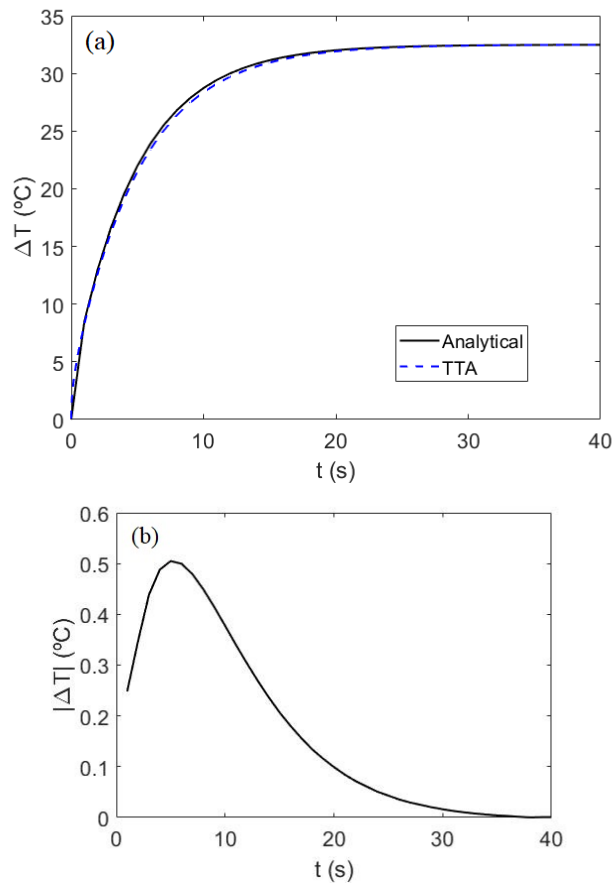

Fig. 9. (a) Average temperature increase at the vacuum side of the wall $(x=0)$ as a function of time. (b) Absolute temperature difference between the analytical and the TTA cases shown in (a).
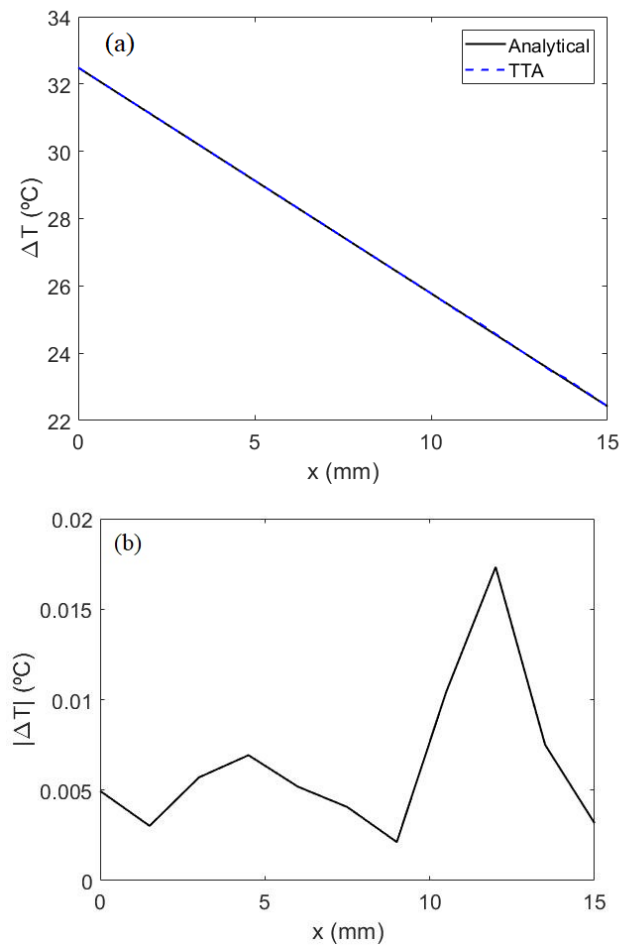

Fig. 10. (a) Average temperature increase along the wall at the steady state $(t=40 \mathrm{~s})$. (b) Absolute temperature difference between the analytical and the TTA cases shown in (a).

to get 1000 eigenvalues in $0.43 \mathrm{~s}$. Then, a convergence test of the analytical solution (given by eq. (16)) was carried out by considering different number of terms in the summation. It was found that very few summation terms were needed to reach the convergence for this particular problem. In fact, the maximum absolute difference in the temperature between 1 and 10 terms is $6 \times 10^{-3}{ }^{\circ} \mathrm{C}$. Similarly, the maximum absolute difference in the temperature between the cases with 2 and 10 terms is $2.6 \times 10^{-10}{ }^{\circ} \mathrm{C}$. Thus, for this particular case, the summation convergence is reached with just the first two terms. Once the required number of eigenvalues are known, the computational time required to calculate the analytical summation given by eq. (16) is $0.3 \mathrm{~s}$. In total, the computational time required to obtain the data depicted in the figures of Example IV is approximately $1 \mathrm{~s}$. From these results it is shown the huge improvement in terms of computational time achieved when using the analytical model instead of the TTA numerical simulations.

\section{THERMAL ANALYSIS OF AN RF ELECTRON GUN PHOTOINJECTOR}

The purpose of this Section is to analyse the suitability of applying the 1D theoretical expressions (derived in Section II) in order to obtain a first approximation of the temperature increase caused by the RF pulse heating phenomenon in $3 \mathrm{D}$ cavities which are part of RF accelerating systems. In Section II the temperature increase in a 1D metallic wall was studied by considering separately two effects due to the RF pulse heating. First, the sharp temperature increase occurring during the RF pulse very close to the vacuum side of the wall. Second, the average temperature increase in the wall after many RF pulses are driven into the structure, which finally evolves to a steady state case with a spatial temperature distribution along the wall.

In the first case, the pulse duration in typical RF accelerating devices is too short to allow effective thermal diffusion across the wall, as it was already discussed in Example II of Section II. Therefore, the sharp temperature increase during the RF pulse is a phenomenon depending only on the electromagnetic field intensity at the local point of the surface. According to this, the 3D details of the device (including the cooling system) can be neglected in the analysis. And consequently, the 1D theoretical model presented in Subsection II-A is expected to give good results for realistic $3 \mathrm{D}$ devices.

In the second case, for the temperature increase at the steady state after many RF pulses, the 3D details of the geometry of the device might play an important role in the final temperature distribution. Hence, further investigation must be carried out for this case in order to evaluate the goodness of using the 1D theoretical expressions to approximate the RF pulse heating phenomenon in realistic $3 \mathrm{D}$ devices. To reach this aim, the average temperature increase at the steady state is analysed for an RF electron gun accelerating cavity, comparing the results obtained with the TTA numerical simulations and the predictions of the $1 \mathrm{D}$ theoretical expressions.

The geometry of the device that we have studied is shown in Fig. 11, which is similar to other RF electron guns developed for X-band operation [15], [16]. It consists of six accelerating cavities, each of them with length $\lambda / 2$ (being $\lambda$ the wavelength of the RF electromagnetic wave in free space), except the first 
one which is 0.6 times the length of the others. The present $\mathrm{RF}$ gun is designed to operate in SW mode with the $\pi$-mode advance per cell operating at a frequency of $f=11.994 \mathrm{GHz}$. The RF gun is connected to the RF generator external circuit by means of a coaxial coupler. The filling time of the RF gun is $\tau=\frac{2 Q_{L}}{\omega}=112.5 \mathrm{~ns}, Q_{L}$ being the loaded quality factor of the RF gun cavity. The gun is intended to operate in pulsed mode with an RF pulse duration of $t_{\text {on }}=400 \mathrm{~ns}$ and $f_{p}=400 \mathrm{~Hz}$ repetition rate. The RF electric field profile along the RF electron gun axis after the filling transient is depicted in Fig. 12. As it can be noticed, the magnitude of the RF electric field is almost the same for all the cavities despite the first cathode half-cell has a geometry different from the others. This regular RF electric field in each cavity indicates that the energy stored in each cavity is mostly the same. Taking this fact into account, the thermal analysis of the photoinjector can be reduced to the study of one single full cell. This assumption greatly diminishes the computational requirements and time needed to perform the TTA thermal simulations. Therefore, we will focus on the thermal analysis of the full cells and disregard the analysis of the half-cell since its complex geometry cannot be studied with a $1 \mathrm{D}$ model. As we will see next, this assumption is reasonable since the results between methods are compatible.

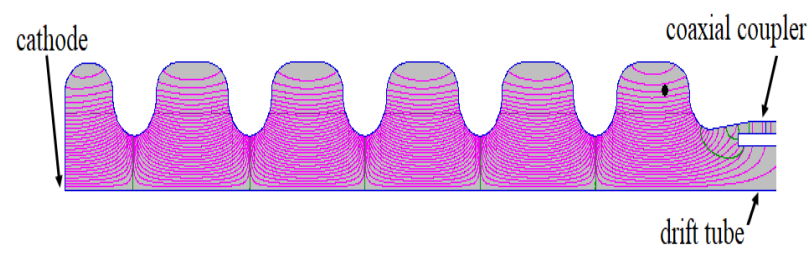

Fig. 11. Scheme of a 5.6 cell RF electron gun photoinjector.

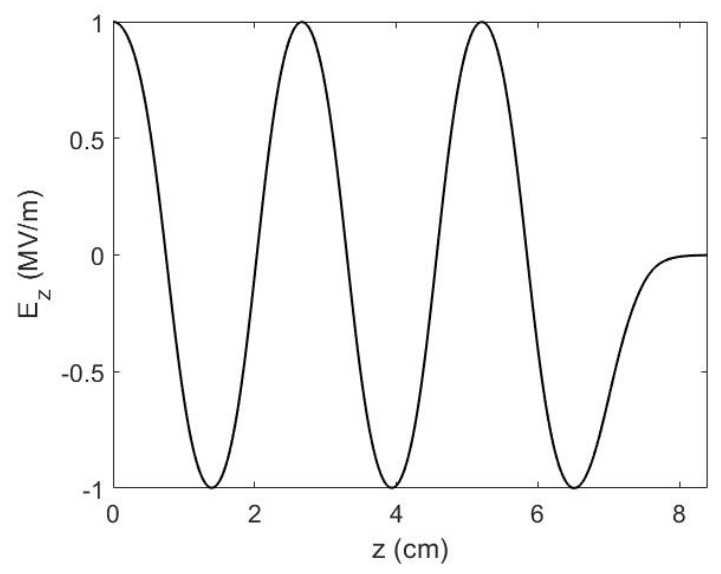

Fig. 12. Axial RF electric field along the RF electron gun photoinjector axis (normalized to $1 \mathrm{MV} / \mathrm{m}$ at cathode).

To proceed with such simulations, firstly the surface losses due to the RF electromagnetic field in the gun cavity are computed with the software Ansys HFSS. In Fig. 13 the surface losses are depicted for the case where the RF electron gun is excited with a $200 \mathrm{MV} / \mathrm{m}$ cathode field. Then, the surface losses are exported from HFSS to TTA in order to perform the thermal simulations. It is worth mentioning that the surface losses provided by HFSS constitute the average power lost per unit area assuming a harmonic constant amplitude signal. Since in the case of the photoinjector there will be a transient, the values provided by HFSS must be scaled to consider accurately the average power losses. Recalling eq. (14), the magnetic field amplitude along the cavity walls can be decomposed as $H_{\|, 0}(t)=H_{\|, 0} F(t)$, being $F(t)$ the function that contains the temporal variation due to the transient. Thus, to take into account the transient in TTA, the surface loss density imported from HFSS, $P_{H F S S}$, must be scaled when it is read in TTA in the following way:

$$
P_{T T A}=\left(f_{p} \int_{0}^{T_{p}}[F(t)]^{2} d t\right) P_{H F S S}
$$

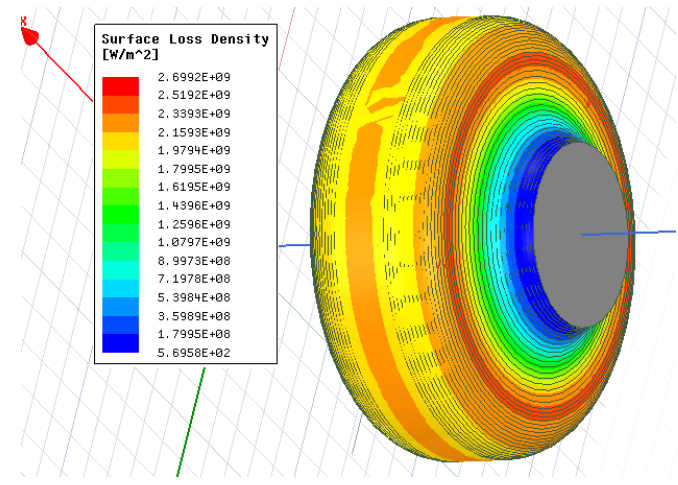

Fig. 13. Surface loss density in the walls of the cavity computed with HFSS for a cathode field of $200 \mathrm{MV} / \mathrm{m}$.

Furthermore, the scheme of the photoinjector cavity in TTA is depicted in Fig. 14. The cavity radius is $R_{c}=$ $11.029 \mathrm{~mm}$ and the cavity length is $L_{\text {cav }}=12.498 \mathrm{~mm}$. The presence of a water cooling channel has been considered, with square section $(a=10 \mathrm{~mm})$, heat transfer coefficient $h=1.2 \times 10^{4} \mathrm{Wm}^{-2} \mathrm{~K}^{-1}$, and fluid temperature $T_{\infty}=0^{\circ} \mathrm{C}$. The separation between the top of the cavity and the lower side of the cooling channel is $L=15 \mathrm{~mm}$. The material for the bulk of the photoinjector is copper, with the same properties as described in the Example I. The initial temperature is assumed to be uniform for all the cavity bulk, $T_{0}=0^{\circ} \mathrm{C}$.

The temperature distribution in the RF electron gun cavity after the steady state has been reached is shown in Fig. 15. When the RF gun is operated with a cathode field of $200 \mathrm{MV} / \mathrm{m}$, the maximum temperature increase found along the cavity wall is $T_{\max }=11.9^{\circ} \mathrm{C}$.

Now, it remains to estimate the maximum temperature increase at the steady state in the photoinjector cavity using the corresponding analytical expression of the 1D model (recall the eq. (18)). Two aspects must be taken into consideration to explore properly this $1 \mathrm{D}$ formula to the case of the $3 \mathrm{D}$ cavity. First, an equivalent heat transfer coefficient $h_{e q}$ must be chosen for the 1D model in order to compensate the possible difference between the area size of the pillbox inner surface, $A_{\text {heat }}$, where the heat sources are located; and the area of the water cooling channel, $A_{\text {cooling, }}$, where the heat transfer due 


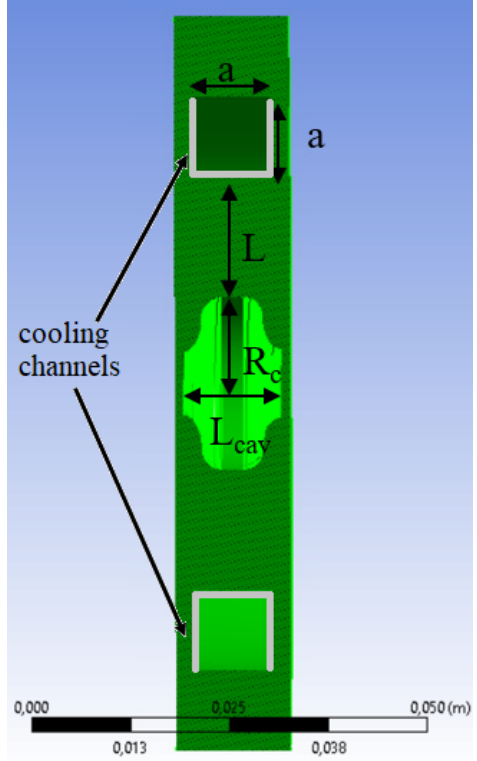

Fig. 14. Scheme of the RF gun cavity with water cooling channel analyzed with TTA

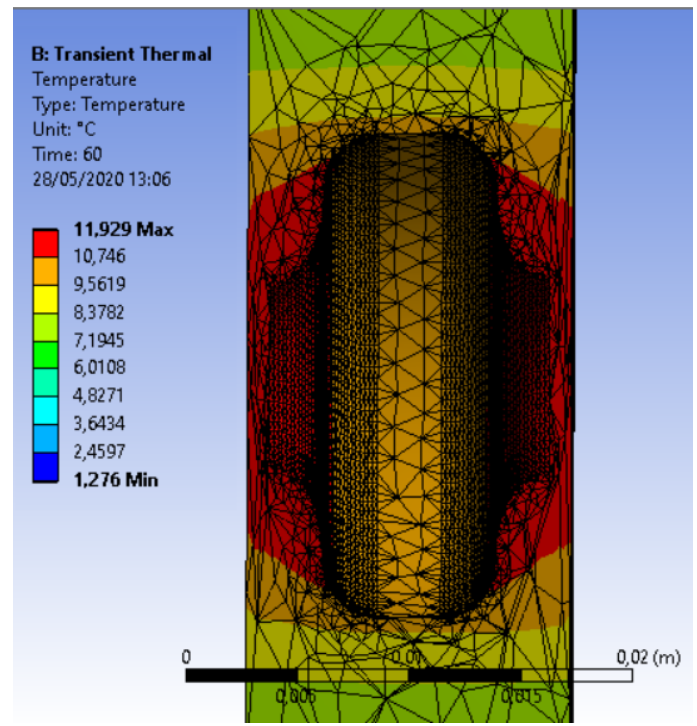

Fig. 15. Temperature distribution in the RF electron gun cavity at the steady state (cooling channel is out of the frame); results from TTA when there is a cathode electric field of $200 \mathrm{MV} / \mathrm{m}$.

to the convection phenomenon occurs. In the $1 \mathrm{D}$ model, it is implicitly assumed that the vacuum surface (where the heat sources are) and the cooling system surface have the same area. As a general rule, for 3D devices, it is not expected that both areas are equal. To adjust this mismatch, we propose to take in the 1D model an equivalent heat transfer coefficient defined as:

$$
h_{\text {eq }}=\frac{A_{\text {cooling }}}{A_{\text {heat }}} h
$$

where $h$ represents the heat transfer coefficient of the water cooling channel of the 3D structure. Regarding the area of the water cooling channel, $A_{\text {cooling }}$, only the area corresponding to the bottom and the lateral faces (neglecting the top face) is taken into account. In Fig. 14 those surfaces are marked in grey. According to this, the area of cooling is given by

$$
A_{\text {cooling }}=2 \pi\left(L+R_{c}\right) a+2 \pi\left[\left(L+R_{c}+a\right)^{2}-\left(L+R_{c}\right)^{2}\right]
$$

The physical reason that motivates neglecting the top face is that this surface has no direct line of sight with the heat sources and hence no significant effects in the cooling are expected. This choice might seem a rough approximation but in fact gives satisfactory results as we will see next. For the particular case of this example, $h_{e q}=5.087 h$.

The second consideration is related to the fact that in the gun cavity the surface losses are not uniform, whilst the 1D model assumes uniform power losses per unit area, $q_{s, 0}$. Consequently, a spatial average power losses per unit area coefficient $q_{s, 0, \text { avg }}$ has to be defined and employed in eq. (18):

$$
q_{s, 0, a v g}=\frac{P_{\text {loss }}}{A_{\text {heating }}}
$$

where $P_{\text {loss }}$ is the total RF power lost by Joule effect in the walls of the cavity, and $A_{\text {heating }}$ is the area of the cavity in contact with the RF electromagnetic fields. The value of $P_{\text {loss }}$ can be calculated using either HFSS or other RF electromagnetic codes. For example, the free distribution software SUPERFISH [17] would be a good option for such purpose.

In Fig. 16 the maximum temperature increase in the RF gun cavity at the steady state is depicted for different values of the RF electric field amplitude at the photoinjector cathode. The results of both TTA simulations and the 1D model are included for comparison. With respect to the chi-squared test, it is obtained $\tilde{\chi}_{0}^{2}=0.01$ and $P\left(\tilde{\chi}^{2} \geqslant \tilde{\chi}_{0}^{2}\right) \approx 100 \%$. Besides, it is observed that the maximum absolute difference between the TTA results and the analytical approximation is always below $0.6^{\circ} \mathrm{C}$ or, in terms of the relative difference, below $6 \%$. Note that the relative difference is defined as $D(\%)=100 \times\left(T_{T T A}-T_{\text {analytical }}\right) / T_{T T A}$, where $T_{T T A}$ and $T_{\text {analytical }}$ are the temperatures obtained with TTA and the 1D analytical model, respectively. For many practical applications that require the thermal analysis of an RF accelerating structure, like the assessment of thermal stresses that can cause surface roughening and microcracks, the design of the cooling system, and the RF breakdown risk, the current values of accuracy found are good enough. For example, in order to prevent high RF breakdown rates, in the technical literature it is recommended that the maximum temperature increase is maintained below the safe limit of $60^{\circ} \mathrm{C}$ [18], or $50^{\circ} \mathrm{C}$ according to other authors [19]. Therefore, an approximation that gives the maximum temperature increase in the device with a maximum difference between the real value and the approximation of only one or two Celsius degrees, is accurate enough for the purpose of assessing the design of RF accelerating structures aiming to keep a low RF breakdown rate. It is worth mentioning that in [18], the thermal analysis of a SW RF accelerating cavity is performed using the approximate expression of the 1D model described in [7]. This approximation from [7] is found to have differences with the accurate expression of the $1 \mathrm{D}$ model up to $1.5^{\circ} \mathrm{C}$ (recall 
Fig. 8(a)) and, despite this, it is employed for the design of a $3 \mathrm{D}$ realistic RF accelerating component. Another example of the usefulness of the analytical approximations provided in this paper would be the analysis of the thermal stresses caused by the RF pulse heating. Indeed, in [20] a theoretical model to analyse the thermal fatigue in the material is developed by using the temperature increase approximation described in [7]. This fact also supports the statement that the accuracy of the theoretical approximations presented in this paper is good enough for practical applications.

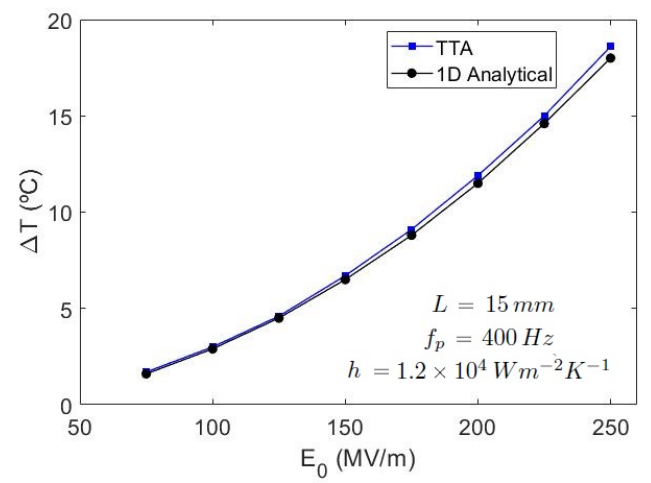

Fig. 16. Maximum temperature increase in the RF gun at the steady state for different values of electric field amplitude at cathode. Comparison between the TTA simulations and the results of the $1 \mathrm{D}$ model.

For completeness, the comparison between the TTA simulations and the 1D model is performed for other working points of the RF cavity. In Fig. 17, the temperature increase for the RF cavity is shown with the same parameters, with the exception of $f_{p}=200 \mathrm{~Hz}$ and $h=0.5 \times 10^{4} \mathrm{Wm}^{-2} \mathrm{~K}^{-1}$. For this case, the chi-squared statistical parameters are $\tilde{\chi}_{0}^{2}=0.012$ and $P\left(\tilde{\chi}^{2} \geqslant \tilde{\chi}_{0}^{2}\right) \approx 100 \%$. Besides, the maximum absolute temperature difference is $0.6^{\circ} \mathrm{C}$, which in terms of relative difference is below the $5 \%$. Similarly, in Fig. 18 the results for $f_{p}=1000 \mathrm{~Hz}, h=1.0 \times 10^{4} \mathrm{Wm}^{-2} \mathrm{~K}^{-1}$, and $L=25 \mathrm{~mm}$ are depicted. For this case, it is found $\tilde{\chi}_{0}^{2}=0.097$ and $P\left(\tilde{\chi}^{2} \geqslant \tilde{\chi}_{0}^{2}\right)=99.85 \%$. The maximum absolute temperature difference of $2.5^{\circ} \mathrm{C}$, which in terms of relative difference is below the $6 \%$. Once again, the temperature difference between the analytical approximation and the TTA results are within the range of usefulness for practical applications.

Finally, it is interesting to compare the computational times required by the $1 \mathrm{D}$ analytical analysis and the TTA numerical simulations. The following results belong to an Intel(R) Core(TM) i7- 865U CPU @ $2.10 \mathrm{GHz}, 16$ GB RAM. With regard to the TTA simulations, firstly it is required to compute the power losses with HFSS. This step has to be performed only once for each device and, for this geometry, the HFSS analysis took 5 minutes. Then, a TTA simulation must be carried out to obtain the temperature increase for each value of the RF electric field amplitude $E_{0}$ shown in Figs. 16-18. The computational time for each of these TTA simulations is approximately 7 minutes. Hence, the total time to generate the data required for Figs. 16-18, which have a total of 24 points, is 173 minutes, i.e., approximately 2 hours and 53 minutes. With regard to the $1 \mathrm{D}$ analytical analysis, a total computational time

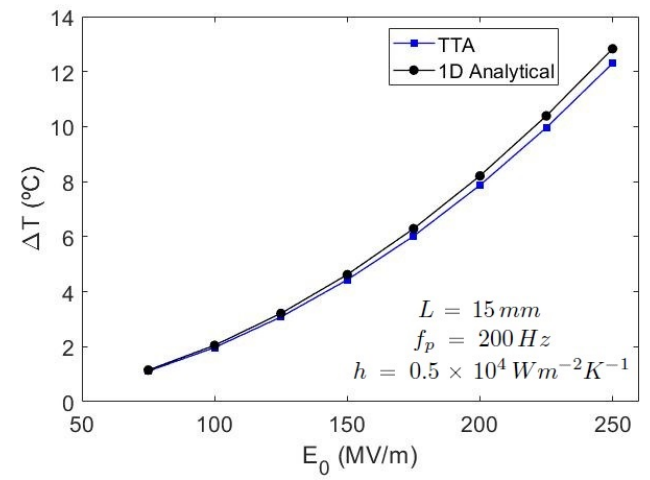

Fig. 17. Maximum temperature increase in the RF gun at the steady state for different values of electric field amplitude at cathode. Comparison between the TTA simulations and the results of the $1 \mathrm{D}$ model.

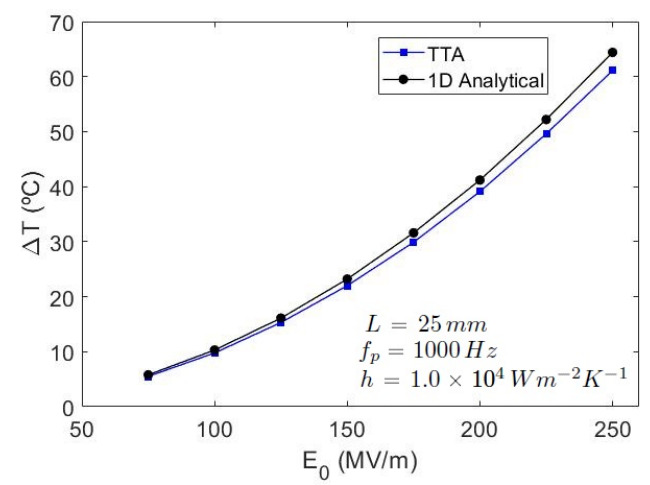

Fig. 18. Maximum temperature increase in the RF gun at the steady state for different values of electric field amplitude at cathode. Comparison between the TTA simulations and the results of the 1D model.

of $0.004 \mathrm{~s}$ was required to calculate the temperature increase of the 24 points corresponding to Figs. 16-18. Thus, the 1D analytical analysis is found to be more than two million times faster than the TTA numerical simulations, evidencing the usefulness of this method to obtain a fast approximation of the maximum temperature increase for the RF photoinjector cavity.

\section{THERMAL ANALYSIS OF A LINAC CAVITY}

In this Section we perform the thermal analysis of a Linac structure both using the 1D theoretical expressions and the TTA numerical simulations. The procedure will be very similar to that described above for the study of the RF photoinjector cavity. Similarly, the objective is to obtain the maximum average temperature increase along the device surface at the steady state.

The Linac structure under analysis is composed of 108 accelerating cells operating in the Travelling Wave (TW) configuration at X-band [21]. The geometry of the accelerating cells is shown in Fig. 19, where three of them are depicted. The thermal analysis will be carried out for one single of these cavities. The phase advance between neighbour cavities is $2 \pi / 3$ and the RF frequency is $f=11.994 \mathrm{GHz}$. The Linac structure is excited in pulsed mode with rectangular RF pulses 
with duration $t_{o n}=400 \mathrm{~ns}$ and $f_{p}=400 \mathrm{~Hz}$ repetition rate. The RF electric field profile of one single of the linac cavities is shown in Fig. 20.

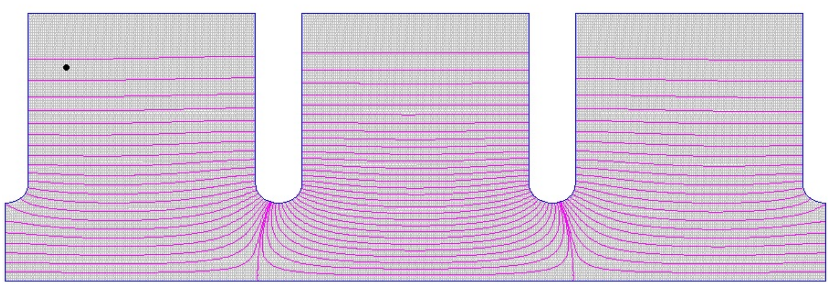

Fig. 19. Partial view of the Linac accelerating structure (three cells are shown).

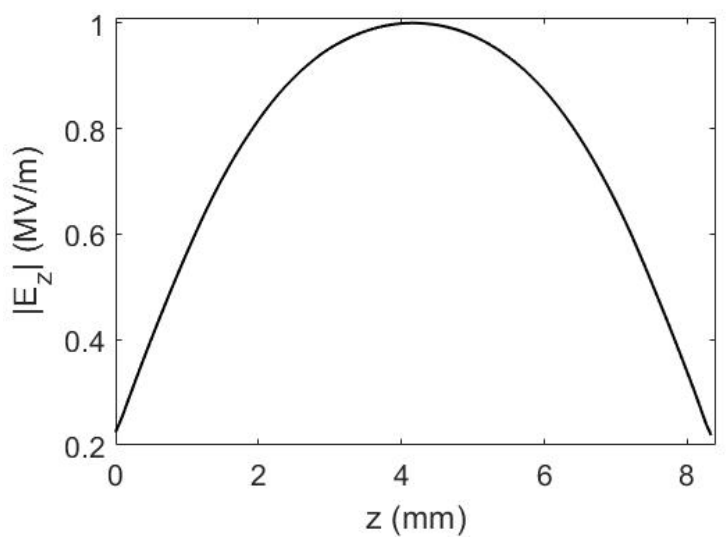

Fig. 20. Modulus of the axial RF electric field along the linac cavity (normalized for $1 \mathrm{MV} / \mathrm{m}$ at cathode).

Similarly to the RF photoinjector case, the RF power losses along the device surfaces are computed with HFSS and exported to TTA for the thermal analysis. The scheme of the linac cavity in TTA is depicted in Fig. 21. The cavity radius is $R_{c}=9.869 \mathrm{~mm}$ and the cavity length is $L_{c a v}=8.333 \mathrm{~mm}$. It has been considered the presence of a water cooling channel, with square section $(a=6.933 \mathrm{~mm})$, heat transfer coefficient $h=1.0 \times 10^{4} \mathrm{Wm}^{-2} \mathrm{~K}^{-1}$, and fluid temperature $T_{\infty}=0^{\circ} \mathrm{C}$. In the scheme, the cooling channel surfaces that are accounted for the calculation of $h_{e q}$ (recall that this parameter is required for the 1D model temperature increase estimation) are marked in green. The same criterion as in the photoinjector case was taken, leading to $h_{e q}=3.297 \mathrm{~h}$. The separation between the top of the cavity and the bottom of the cooling channel is $L=15 \mathrm{~mm}$. The material for the bulk of the cavity is copper, with the same properties as described in the Example I. The initial temperature is assumed to be uniform for all the cavity bulk, $T_{0}=0^{\circ} \mathrm{C}$.

In Fig. 22 the maximum temperature increase in the linac cavity at the steady state is depicted for different values of the RF electric field amplitude. The results of both TTA simulations and the 1D model are included for comparison. Regarding the chi-squared statistics, it is found $\tilde{\chi}_{0}^{2}=0.012$ and $P\left(\tilde{\chi}^{2} \geqslant \tilde{\chi}_{0}^{2}\right) \approx 100 \%$. The maximum absolute difference between the TTA results and the analytical approximation is

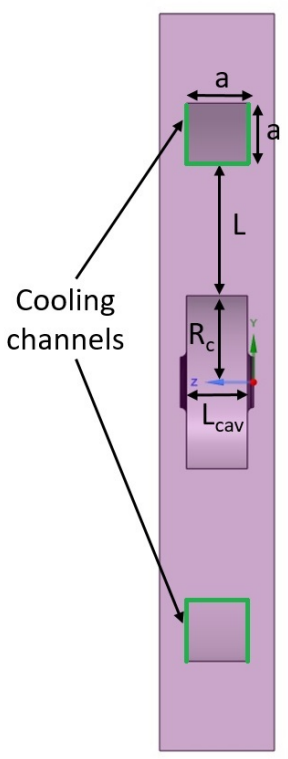

Fig. 21. Scheme of the linac cavity with water cooling channel analyzed with TTA.

always below $0.65^{\circ} \mathrm{C}$ or, in terms of the relative difference, below $4 \%$.

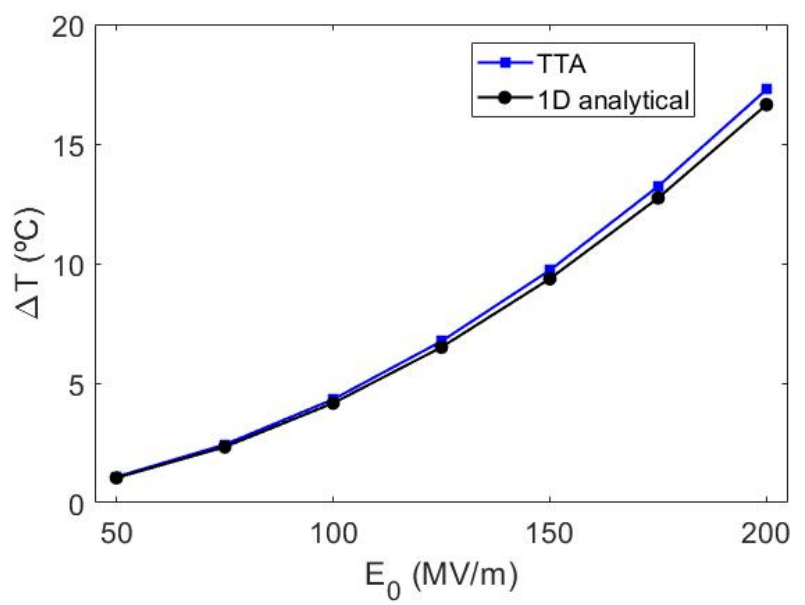

Fig. 22. Maximum temperature increase in the linac cavity at the steady state for different values of the RF electric field amplitude. Comparison between the TTA simulations and the results of the 1D model.

Concerning to the computational time, very similar results to the previous example for the RF photoinjector were found. The HFSS analysis took 6 minutes, whilst each TTA simulation for a certain value of the RF electric field amplitude $E_{0}$ lasted 7 minutes. For the 1D analytical analysis, a total computational time below $0.004 s$ was required to calculate the temperature increase for the seven different values of the RF electric field amplitude $E_{0}$. Thus, it is clear again the usefulness of the 1D analytical method to obtain a quick approximation for the maximum temperature increase in the device. 


\section{Conclusions}

In this paper, the temperature increase due to the RF pulse heating phenomenon has been analysed in RF accelerator structures. For this purpose, the 1D heat transfer differential equation has been solved in a thick metallic slab for certain specific cases, allowing to obtain simple analytic mathematical expressions. The peak temperature increase during the pulse is obtained for the cases of a square RF pulse and an RF pulse with transient. Similarly, the average temperature increase after many RF pulses is also provided. In the latter case, the theoretical model assumes that the device exchanges heat with the environment by means of the convection mechanism. This allows us to consider the interesting case of a cooling system based on a water turbulent flow through a pipe.

The 1D analytical expressions for the temperature increase in the slab presented in this paper are aimed at improving the design procedure of RF accelerating cavities. This is accomplished by reducing the computational time required to perform the thermal analysis with regard to using of 3D FEM codes. For instance, as it is discussed throughout the paper, the computational time required to analyse the temperature increase during the RF pulse can be accelerated up to 200 times with respect to the Ansys Fluent numerical simulations, for a standard PC (Intel Core i5, $3.40 \mathrm{GHz}, 8 \mathrm{~GB}$ RAM). Thus, an analysis that Ansys Fluent would require a bit more than half an hour can be reduced to only seven seconds. This time reduction allows us to explore the effect of a wide range of parameters in the thermal behaviour of the device in very short times compared with a numerical analysis. For instance, this is useful for the optimization of the geometry and dimensions of a cavity in order to reduce as much as possible the temperature increase due to RF pulsed heating during its operation.

It is worth mentioning that, for many practical applications that motivate the thermal analysis of an RF accelerating structure, the accuracy achieved with the 1D analytical expressions is good enough when comparing with the accurate 3D numerical simulations performed with Ansys Fluent or Ansys Thermal Transient. Some examples of the applications that could be analysed using the 1D analytical model are the assessment of thermal stresses that can cause surface roughening and microcracks, the design of the cooling system, and the analysis of the RF breakdown risk.

In this paper, two realistic 3D structures have been analysed both with the 1D analytical formulas and the Ansys numerical simulations. These two devices are an RF photoinjector cavity, and a linac accelerating cavity. In both cases the results evidence that the $1 \mathrm{D}$ analytical formulas provide an useful approximation to the temperature increase in the devices. However, it must be remarked that the method described in this manuscript is intended to provide a quick approximation to the temperature increase in the device, but of course it will not be as accurate as proper $3 \mathrm{D}$ numerical simulations.

\section{ACKNOWLEDGEMENTS}

This work was supported by the European Union's Horizon 2020 research and innovation programme under the grant agreement No 777431 (XLS CompactLight).
This research was also supported by the Valencian Regional Government VALi+D postdoctoral grant (APOSTD/2019/155).

\section{REFERENCES}

[1] V. Dolgashev, S. Tantawi, Y. Higashi, T. Higo, Proceedings of the 11th European Particle Accelerator Conference, "Status of high power tests of normal conducting single-cell structures", pp. 742-744, Genoa, 2008.

[2] X. Lin, H. Zha, J. Shi, H. Chen, X. Wu, Z. Liu, Proceedings of the 10th International Particle Accelerator Conference (IPAC2019), "Analysis and simulation of the after-pulse RF breakdown", pp. 196-199, Melbourne, 2019.

[3] www.ansys.com

[4] www.3ds.com/products-services/simulia

[5] www.temf.tu-darmstadt.de/forschung_5/fitmafia/fit.en.jsp

[6] D. B. Davidson, " Computational Electromagnetics for RF and Microwave Engineering", 2nd edition, Cambridge University Press, 2011.

[7] D. P. Pritzkau, "RF Pulsed Heating", Ph. D. Dissertation Stanford University, 2001.

[8] www.ansys.com/products/fluids/ansys-fluent

[9] J. D. Jackson, Classical Electrodynamics, 3rd edition, John Wiley \& Sons Inc., New York, NY, 1999.

[10] M. C. Wendl, Theoretical Foundations of Conduction and Convection Heat Transfer, The Wendl Foundation, Saint Louis, U.S.A., 2012.

[11] T. P. Wangler, RF Linear Accelerators, 2nd edition, WILEY-VCH Verlag $\mathrm{GmbH} \&$ Co. KGaA, Weinheim, 2008.

[12] G. Arfken, "Mathematical methods por phsysicists", Third Edition, Academic Press Inc., 1985.

[13] D. González-Iglesias, A. Aksoy, D. Esperante, B. Gimeno, M. Boronat, C. Blanch, J. Fuster, "X-band RF photoinjector design for the XLSCompactLight project", to be submitted for publication.

[14] J. Taylor, "An Introduction to Error Analysis : the Study of Uncertainties In Physical Measurements", ed. Sausalito, 2nd edition, University Science Books, California, 1997.

[15] A.E. Vlieks, V. Dolgashev, S. Tantawi, S. Anderson, F. Hartemann, R. Marsh, "X-band RF gun development", pp. 3816-3818, Proceedings of IPAC-10, Kyoto, Japan.

[16] R. Marsh, "Modeling and design of an X-band rf photoinjector", Physical Review Accelerators and Beams, vol. 15, pp. 102001, 2012.

[17] K. Halbach and R. F. Holsiger, "SUPERFISH - A Computer Program for Evaluation of RF Cavities with Cylindrical Symmetry", Particle Accelerators, vol. 7, pp. 213-222, 1976

[18] M. Behtouei, "Design and Measurements of the High Gradient Accelerating Structures", PhD. Thesis, University of Rome "La Sapienza", 2019, Italy.

[19] Private communication with the Prof. Dr. Avni Aksoy from the University of Ankara, Turkey.

[20] S. Kuzikov, M. Plotkin, "Theory of Thermal Fatigue Caused by RF Pulsed Heating", International Journal of Infrared and Millimeter Waves, vol. 29, pp. 298-311, 2008.

[21] Courtesy of the XLS-CompactLight project collaboration. (https://www.compactlight.eu/Main/HomePage) 